\title{
Reconstrução da estrutura facial por biomateriais: revisão de literatura
}

\section{Facial structure reconstruction by biomaterials: literature review}

\author{
MÁrio MAIA ${ }^{1}$ \\ Emília Silva KLein ${ }^{2}$ \\ Tatiana Verastegui Monje 3 \\ Carlos Pagliosa ${ }^{4}$
}

Trabalho realizado na Clínica de Cirurgia Plástica do Hospital Belo Horizonte, Belo Horizonte, MG, Brasil.

Artigo submetido pelo SGP (Sistema de Gestão de Publicações) da RBCP.

Artigo recebido: 21/6/2009 Artigo aceito: $12 / 5 / 2010$

\begin{abstract}
RESUMO
A região facial apresenta um desafio único para o implante de biomateriais, porque o esforço de tração dos músculos dessa região produz carregamento variável em diferentes regiões. Este trabalho apresenta as causas do amplo uso de biomateriais na reconstrução facial, descrevendo as características, as vantagens e as desvantagens de cada tipo específico de material para cada região da face.
\end{abstract}

Descritores: Materiais biocompatíveis. Face. Substitutos ósseos.

\section{SUMMARY}

The facial region presents an unique challenge for implantable biomaterials because the pull of the facial muscles produces variable loading in different regions. This work presents the reasons of the wide use of biomaterial in facial reconstruction, pointing out the advantages and the disadvantages of each specific material type for each face region.

Descriptors: Biocompatible materials. Face. Bone substitutes.

\section{INTRODUÇÃO}

Os biomateriais são utilizados rotineiramente em aplicações médicas, tais como distribuição de drogas, engenharia de tecidos, dispositivos para terapias e como contrastes em diagnóstico por imagens ${ }^{1}$. Tem sido reconhecido que as propriedades dos materiais afetam os resultados biológicos e podem ter impacto profundo na proliferação das células e no remodelamento dos tecidos. Portanto, a questão central das pesquisas biomédicas está em projetar e controlar as propriedades dos materiais para conseguir uma resposta biológica específica² ${ }^{2}$.

Uma definição amplamente aceita sobre biomateriais é qualquer material, natural ou artificial, que compreende o todo ou uma parte de uma estrutura viva ou um dispositivo biomédico que executa, acrescenta ou substitui uma função natural $^{3}$.

Uma das vantagens em se utilizar materiais sintéticos é evitar a coleta de outros materiais autogênicos para enxerto, como ossos, músculos e gordura. O tempo total necessário para a cirurgia é reduzido e também a extensão da ferida cirúrgica. Na clínica, a necessidade de enxertos ósseos vem crescendo em decorrência das cirurgias por traumas, tumores, craniossinostose e cirurgias com finalidades estéticas. A utilização de materiais sintéticos evita o uso de enxertos com tecidos alogênicos a partir de um banco de tecidos. Outra vantagem associada aos biomateriais é que são fabricados sob condições controladas e suas composições químicas são bem conhecidas e os materiais estão disponíveis em qualquer tempo e quantidade, considerando que as grandes reconstruções ou reoperações podem limitar o uso de enxertos autogênicos, evitando a morbidade dos doadores, a deformidade de crescimento, as dores e a reabsorção $0^{4-8}$.

A seleção dos materiais adequados ao desenvolvimento de implantes requer cumprir alguns critérios, cujos efeitos sobre o corpo humano têm que ser o mínimo possível. Os

1. Membro Especialista da Sociedade Brasileira de Cirurgia Plástica (SBCP), Membro da Clínica de Cirurgia Plástica e Preceptor da Pós-Graduação Médica em Cirurgia Plástica do Hospital Belo Horizonte, Belo Horizonte, MG, Brasil.

2. Membro Titular da SBCP; Coordenadora da Clínica de Cirurgia Plástica e Preceptora da Pós-Graduação Médica em Cirurgia Plástica do Hospital Belo Horizonte, Belo Horizonte, MG, Brasil.

3. Membro Especialista da SBCP.

4. Pós-doutor; Consultor. 
fatores de maior relevância devem ser estudados em detalhes, tais como': biocompatibilidade, biodegradabilidade e biorreabsorvibilidade, taxa de degradação, tamanho de poro e morfologia da superfície.

\section{Biocompatibilidade}

A biocompatibilidade desempenha um papel chave, o que garante que os materiais são seguros para uso dentro do corpo humano e nos fluidos endógenos. Os materiais biocompatíveis são definidos como aqueles que não induzem nenhuma resposta inflamatória, devem possuir extrema imunogenicidade ou citotoxicidade para as células nativas, tecidos ou órgãos vivos. Normalmente, os implantes inseridos no corpo humano perduram por um período de tempo e os produtos extras resultantes do processo de degradação não devem produzir nenhum material e/ou elemento nocivo ao organismo.

As propriedades de superfície dos biomateriais desempenham um fator importante para regular a reação do corpo estranho nas primeiras duas a quatro semanas seguidas ao implante. O conhecimento da reação causada pelo corpo estranho pode impactar na biocompatibilidade do dispositivo médico, da prótese ou do biomaterial implantado ${ }^{10}$.

\section{Biodegradabilidade e biorreabsorvibilidade}

Os materiais biodegradáveis, como os polímeros, podem ser decompostos naturalmente e seus produtos permanecerão dentro do corpo humano. Os materiais biorreabsorvíveis são degradados após um período de tempo e os produtos resultantes são atóxicos para efeitos de eliminação gradativa e/ ou pelo metabolismo. A degradação química ocorre de duas formas:

- degradação hidrolítica ou hidrólise - que ocorre simplesmente pela água e;

- degradação enzimática - que ocorre principalmente pela ação de agentes biológicos, tais com as enzimas.

As vantagens do uso de polímeros biodegradáveis em relação aos materiais metálicos tradicionais incluem a redução da capacidade de tensão acumulada, o alívio de dores e a eliminação da necessidade da segunda cirurgia para a remoção dos implantes metálicos ${ }^{10}$.

Taxa de degradação, tamanho de poro e morfologia da superfície

A taxa de degradação do biomaterial implantado deveria coincidir com a taxa de neoformação tecidual, promovendo assim uma transição gradual. Entretanto, a taxa de degradação dos materiais depende de sua composição, nível de pressão e o ambiente nos quais se encontram. A degradação depende diretamente das características da superfície do material, tais como a área superficial e sua porosidade. Grande número de poros é capaz de melhorar o transporte de massa e a neovascularização dentro dos implantes, enquanto poros de diâmetro menores promovem maior razão área por volume. Além do ajuste do tamanho do poro, sua forma também é essencial para a eficiência da regeneração do tecido. Para o crescimento interno do osso, o tamanho de poro ótimo está na faixa de 75 a $250 \mu \mathrm{m}^{10}$.

\section{BIOMATERIAIS NA RECONSTRUÇ̃̃O DA ESTRUTURA FACIAL}

A meta na condução de pacientes com fraturas faciais é a reconstrução da integridade estrutural do esqueleto craniofacial e restaurar sua morfologia. $\mathrm{O}$ esqueleto craniofacial é um sistema complexo com alguns elementos carregados com substancial tensão, enquanto outros componentes têm o propósito de definir a forma. Os elementos que suportam tensão podem ser idealizados como estruturas unidimensionais e serem restauradas por meios unidimensionais: placas. Os elementos que não suportam tensão são tipicamente bidimensionais ou maiores: superfícies planas ou curvas. Juntas elas criam os elementos de estrutura da face. Esse conceito pode ser aplicado para a reconstrução craniofacial póstraumática pelo uso de sistemas de fixação rígidos quando aplicados em regiões de alta pressão. Os sistemas reabsorvíveis são aplicados quando a reconstrução de superfícies bidimensionais ou tridimensionais é de baixa solicitação de carga $^{11}$.

O uso combinado de sistemas reabsorvíveis e não reabsorvíveis em pacientes de trauma facial promove boa estabilização e restauração do contorno sem enxertos de osso cranial e reduz a quantidade de materiais aloplásticos permanentes. Ainda há uma vantagem adicional devido à diminuição de artefatos no diagnóstico por imagens quando tal avaliação se faz necessária.

O uso dos biomateriais para implantes é parte integral da cirurgia facial reconstrutiva e estética. Nesse contexto, a reconstrução facial possui uma ampla variedade de materiais classificados em três grupos:

\section{Cerâmica}

\section{Hidroxiapatita e Cimentos}

Incluem a hidroxiapatita e cimentos de fosfato de cálcio ${ }^{3}$. São excelentes para a reconstrução facial e não impedem a reabsorção de longo prazo, porém, não demonstram significativo crescimento interno ósseo. São recomendados para reconstrução sobre camadas e são projetados para serem osteocondutivos, porém, recrutando osteoblastos do esqueleto adjacente para a reposição óssea ao longo do tempo ${ }^{7}$.

A hidroxiapatita forma o principal componente mineral do osso e constitui $60 \%$ do esqueleto humano calcificado. Sua composição química é $\mathrm{Ca}_{10}\left(\mathrm{PO}_{4}\right)_{6}(\mathrm{OH})_{2}$, sendo a razão cálcio/fósforo de 1,67. A forma sintética da hidroxiapatita 
apresenta similaridade química e cristalográfica, entretanto, não idêntica à hidroxiapatita de ocorrência natural. Esse material cerâmico é sintetizado na forma cristalina em $\mathrm{pH}$ baixo e então sintetizado entre 700 a $1300^{\circ} \mathrm{C}$ para formar uma massa sólida de hidroxiapatita ${ }^{4,8}$. Este tipo de hidroxiapatita sólida está disponível em duas formas, a saber:

- Hidroxiapatita densa: é inteiramente sintética, não apresenta poros e pode ser fabricada em blocos ou grãos. É de difícil conformação e não permite o crescimento interno de tecido. Entretanto, os grânulos apresentam maior adaptabilidade de contorno que os blocos, mas não integridade estrutural intrínseca e não se torna mecanicamente estável até ser envolvida pelo tecido ósteo-fibroso. Os grânulos são difíceis de serem mantidos dentro do local desejado de implante e há possibilidade de migração para áreas não pretendidas após alguns meses ou anos ${ }^{4}$;

- Hidroxiapatita porosa: pode ser produzida sinteticamente ou originada dos esqueletos de corais marinhos. O esqueleto de carbonato de cálcio do coral é convertido quimicamente em hidroxiapatita e a estrutura porosa original do coral é preservada. A vantagem significativa da hidroxiapatita porosa, conforme mostrada na Figura $1 \mathrm{~A}^{12}$, permite o crescimento interno do tecido ósseo e é fixada pelo osso circunjacente em algumas semanas. Por causa da dificuldade de conformação devido à suscetibilidade de fratura, sua aplicação tem sido amplamente utilizada fora das áreas de pressão como enxerto sobre camada ou espaçador em osteotomia facial ${ }^{4}$.

O cimento é a forma não sólida da hidroxiapatita. Esse material é produzido pela direta cristalização da hidroxiapatita em $\mathrm{pH}$ fisiológico e não requer aquecimento para formar um implante estável estruturalmente. O cimento seco é composto de fosfatos dicálcio e tetracálcio, sua cura ocorre em aproximadamente 15 minutos e se converte em hidroxiapatita em 4 horas. Os cimentos são usualmente utilizados para a reconstrução de defeitos faciais extensos. Desenvolvimentos recentes desses cimentos têm produzido

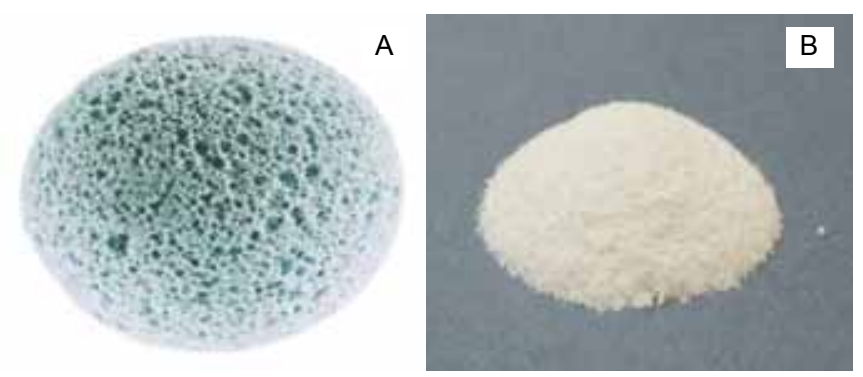

Figura 1-A: hidroxiapatita porosa e B: cimento de hidroxiapatita utilizados para reconstrução da estrutura facial ${ }^{12,13}$. excelentes materiais para serem localmente aplicados com endurecimento in situ ${ }^{7,8}$. A Figura $1 \mathrm{~B}^{13}$ demonstra o cimento de hidroxiapatita.

O espectro das hidroxiapatitas carbonatadas desenvolvidas sobre vidros bioativos está mais próximo da composição do osso do que a hidroxiapatita sintética. A hidroxiapatita densa pode atingir uma resistência a compressão de até $600 \mathrm{MPa}$, mas possui baixa resistência contra fadiga por causa de sua fragilidade. A resistência a compressão decai com a porosidade e com níveis crescentes de fosfato tricálcio no material.

\section{Biomateriais osteoativos}

Os biomateriais osteoativos incluem a matriz de osso desmineralizada e suportes e os vidros bioativos ${ }^{3}$. Esses materiais são caracterizados por serem osteocondutivos e os biovidros são também osteoindutivos, não apresentando falha na interface do osso. Exibem reposição óssea significativa, com menos precisão de contorno e formato que os cimentos e os polímeros.

Os vidros bioativos são materiais baseados na estrutura $\mathrm{SiO}_{2}-\mathrm{Na}_{2} \mathrm{O}-\mathrm{CaO}-\mathrm{P}_{2} \mathrm{O}_{5}-\mathrm{Al}_{2} \mathrm{O}_{3}-\mathrm{MgO}-\mathrm{K}_{2} \mathrm{O}$. Estão disponíveis em pequenos grânulos ou diferentes tipos de blocos, conforme demonstrado na Figura $2^{4}$. Esses materiais sintéticos são quimicamente ligados ao osso. As reações químicas sobre a superfície são baseadas na dissolução, lixiviamento e precipitação de íons. $\mathrm{Na}$ interface de ligação, os osteoblastos fornecem o colágeno, a substância fundamental e as matrizes vesiculares para mineralização primária que resulta em neoformação óssea ${ }^{4}$.

A ligação entre a superfície implantada de vidro imobilizado e os tecidos moles, como os músculos e o subcutâneo, é formada pela anexação das fibras de colágenos em uma camada rica em sílica e contendo hidroxiapatita. Estudos demonstraram que os vidros bioativos são materiais que não favorecem o crescimento de bactérias e a colonização sobre sua superfície. Testes toxicológicos dos vidros bioativos mostraram que esses materiais são aceitos para usos clínicos,
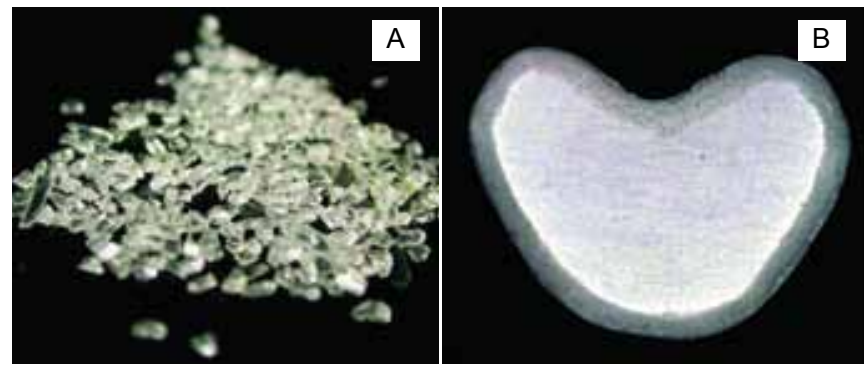

Figura 2-A: grânulos de vidro bioativo e B: placa de vidro bioativo utilizados para reconstrução da estrutura facial ${ }^{4}$. 
tais como reconstruções e cirurgias de trauma e de tumor.

Os biomateriais representam atualmente uma grande opção aos procedimentos cirúrgicos reconstrutivos e obliterativos da região fronto-orbitária. Sinusite frontal supurativa crônica, mucoceles, pioceles, fraturas graves e tumores representam as condições clínicas mais comuns. Osteomielite com necrose do osso frontal e doenças do seio frontal com extensão orbital ou intercranial também podem ser reconstruídas com vidros bioativos ${ }^{4}$.

As placas de vidros bioativos são rígidas e não podem ser moldadas e conformadas pelo cirurgião. A resistência mecânica de compressão é menor para o osso medular, e superior para o osso cortical em relação ao vidro bioativo. Adicionalmente, o vidro bioativo é muito frágil e apresenta baixa tenacidade à fratura. Essas propriedades limitam tanto o uso de vidros bioativos como a hidroxiapatita em áreas submetidas à pressão e em condições clínicas onde o remodelamento do material é obrigatório. A Tabela 1 apresenta uma análise comparativa das propriedades dos ossos medular e cortical comparado com os biovidros e hidroxiapatita densa ${ }^{4}$.

\section{Polímeros}

\section{Polietileno}

O mais comum é o polietileno poroso (Medpor) ${ }^{8,14} \mathrm{e}$ alguns exemplos são apresentados na Figura $3^{15}$. Possui uma estrutura de cadeia linear de carbono e é um dos biomateriais mais utilizados nos últimos 25 anos. É o polímero base para outros materiais, tais como o polipropileno e o politetrafluoretileno e induz um mínimo de reação quando fabricado na forma de alta densidade e alto peso molecular. Esse biomaterial é o padrão de referência como uma substância inerte em termos de reação tecidual.

O polietileno poroso não degrada, não reabsorve em extensão significativa e apresenta a vantagem de permitir o crescimento interno vascular e de tecido mole após uma semana e crescimento interno ósseo após três semanas. É indicado nas reconstruções sobre camadas e entre camadas de segmentos que não estejam sujeitos a pressão. A utilização do polietileno poroso é indicada na reconstrução ou preenchimento da região maxilofacial ${ }^{16}$. É caracterizado por ser não antigênico, antialérgico, inabsorvível, altamente estável, de fácil fixação e disponível em uma grande variedade de formatos para a reconstrução malar, nasal, orbital, do mento e da mandíbula ${ }^{8}$. Seu tamanho de poros varia de 100 a $250 \mu \mathrm{m}$, diferenciando-se de outros materiais, pois esses tamanhos de poros particulares facilitam o crescimento do tecido mole. Dessa forma, o material é estabilizado firmemente na posição desejada.

\section{Polímeros Reabsorvíveis}

Interesse em materiais poliméricos bioabsorvíveis existe há mais de 30 anos. Sua aplicação clínica generalizada é relativamente recente ${ }^{17}$. Inicialmente, foi reconhecido que os polímeros podem ser modelados em dispositivos médicos que poderiam eventualmente ser absorvidos pelo corpo, entretanto, o exato mecanismo ainda é incerto. No início de sua aplicação, muitas complicações foram relatadas com o uso de homopolímeros. Essas complicações incluíam

\begin{tabular}{|c|c|c|c|c|}
\hline & Osso medular & Osso cortical & Vidro bioativo & $\begin{array}{c}\text { Hidroxiapatita } \\
\text { densa }\end{array}$ \\
\hline $\begin{array}{l}\text { Resistência a compressão } \\
\qquad(\mathrm{MPa})\end{array}$ & $2-10$ & $100-230$ & $40-60$ & 600 \\
\hline $\begin{array}{l}\text { Possibilidade clínica de } \\
\text { remodelamento }\end{array}$ & + & + & - & - \\
\hline $\begin{array}{c}\text { Sem morbidade no local } \\
\text { doador }\end{array}$ & - & - & + & + \\
\hline $\begin{array}{c}\text { Disponibilidade em } \\
\text { qualquer quantidade e } \\
\text { tempo }\end{array}$ & \pm & \pm & + & + \\
\hline $\begin{array}{c}\text { Biocompatibilidade } \\
\text { aceitável }\end{array}$ & + & + & + & + \\
\hline $\begin{array}{l}\text { Sem prolongamento do } \\
\text { tempo de operação }\end{array}$ & - & - & + & + \\
\hline Sem reações exotérmicas & + & + & + & + \\
\hline $\begin{array}{c}\text { Propriedade } \\
\text { antimicrobiana }\end{array}$ & - & - & + & + \\
\hline
\end{tabular}

+ significa sim, - significa não e \pm significa incerto. 


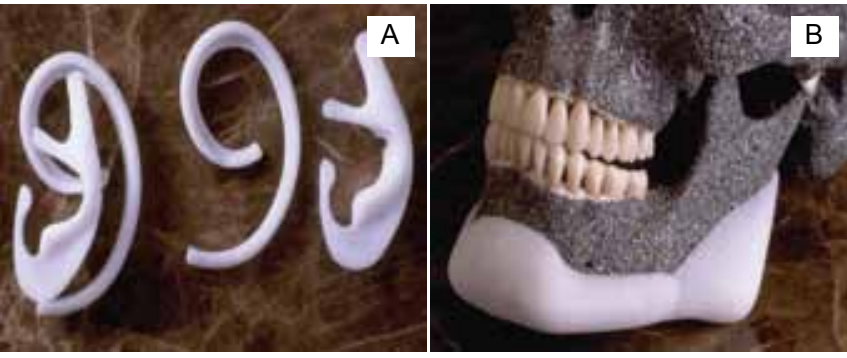

Figura 3-A: implantes de polietileno poroso utilizado na reconstrução auricular e B: implante de polietileno utilizado para implante de queixo ${ }^{15}$.

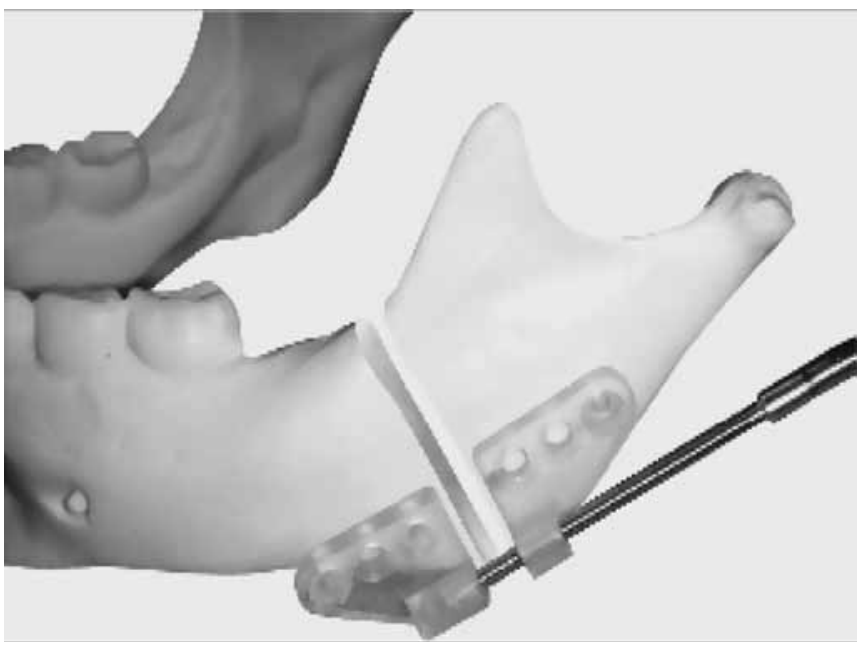

Figura 4 - Implante mandibular de copolímero randômico linear de $82 \%$ de ácido poli-L-lático (PLLA) e 18\% de ácido poligligólico $(P G A)^{17}$.

encapsulamento fibroso, formação de cavidade estéril e uma resposta inflamatória intensa. Menores reações foram observadas com o uso de copolímeros. Esses polímeros prematuramente reabsorvíveis desfrutaram de alguma popularidade, mas não foram amplamente utilizados devido ao longo período de tempo até a reabsorção, a quantidade de inflamação causada e as questões relacionadas à sua estabilidade mecânica.

No final da década de 80 e no início da década de 90 do século passado, o interesse pelo uso de polímeros reabsorvíveis retornou com a mistura de dois diferentes polímeros com características de reabsorção dependentes da proporção da mistura entre ambos. Uma variedade de polímeros reabsorvíveis foi formulada para uso como dispositivos médicos, sendo que o mais estudado é o Lacto Sorb. Consiste de um copolímero randômico linear de $82 \%$ de ácido poli-L-lático (PLLA) e 18\% de ácido poligligólico (PGA), conforme exemplo apresentado na Figura $4{ }^{17}$. Esse composto é reabsorvido durante 12 meses e perde resistência de 3 a 4 meses. Essa característica de reabsorção intermediária permite a reabsorção por hidrólise e fagocitose ${ }^{17}$.

Experimentos com copolímero composto de $82 \%$ de ácido poli-L-lático (PLLA) e 18\% de ácido poligligólico (PGA) mostram-se como uma opção para sistema de fixação reabsorvível e têm sido uma alternativa para os dispositivos de titânio ${ }^{17,18}$. Esse polímero promove uma fixação rígida que permite satisfatório restabelecimento ósseo com a vantagem de ser eliminado pelo corpo. Em comparação com o titânio, as placas de polímeros eram significativamente mais espessas por causa das considerações de resistência e eram moldadas nos mesmos tamanhos e formas que as placas de titânio.

A partir de 2000, foi iniciado o estudo do uso de polímeros reabsorvíveis de único estágio em distração osteogênica craniofacial, principalmente na distração mandibular de neonatos e crianças com sequência de Pierre Robin ${ }^{17}$.

\section{Metais}

Titânio

Nas cirurgias craniofaciais, o titânio é praticamente o único metal utilizado ${ }^{19}$ e pode ser apresentado em diversos dispositivos, conforme mostrados na Figura 5. O sistema de placas de titânio foi o meio mais efetivo e seguro de fixação óssea para a cirurgia de crânio e maxilofacial até 1996. Tradicionalmente, as placas de titânio são de fácil aplicação e bem toleradas pelos pacientes, e os resultados são considerados entre bom a excelente e com baixos índices de complicação pós-operatória ${ }^{20}$. Na população pediátrica acompanhada por mais de dez, a fixação com placas de titânio interfere no crescimento ósseo, exposição da placa, infecção e migração transcranial ${ }^{21}$.

Os dispositivos de titânio usados para fixação rígida são confiáveis para promover resistência e estabilidade do esqueleto ${ }^{18}$. São utilizados há mais de três décadas, principalmente para a fixação de auxílio do osso ancorado do ouvido e epíteses craniofacial. Implantes de titânio osteointegrados podem ocorrer em aplicações de fraturas de mandíbula ${ }^{22}$. É importante que os implantes recuperados de titânio possam ser avaliados para entender melhor sobre o processo de osteointegração e assim correlacionaros diferentes resultados clínicos com as constatações histológicas. Em implantes com bom contato osso-metal, a percentagem do contato do osso com o metal cresce com o tempo. Ao contrário, quando esse contato é pobre, as zonas de tecido mole são mais extensas e são observadas, ocasionalmente, reações de pele.

Os instrumentos de titânio podem ser divididos em duas classes principais: placas e parafusos utilizados em osteossínteses e implante de raiz dentária ${ }^{5}$. A osteointegração dos dispositivos de titânio depende das propriedades de superfície para a relação entre implantes e tecidos. Estudos 


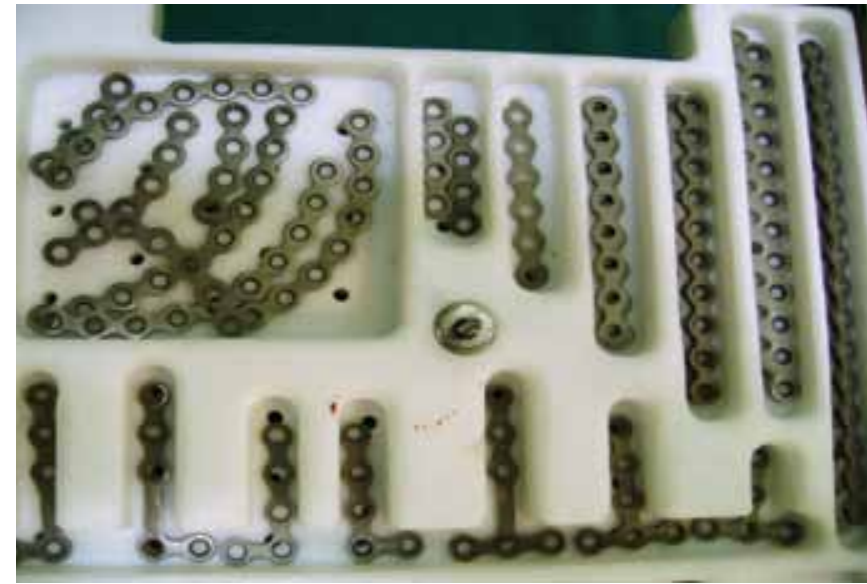

Figura 5 - Dispositivos de titânio utilizados para reconstrução da estrutura facial.

preliminares ${ }^{17}$ provaram que uma camada de $5 \mathrm{~nm}$ de óxido de titânio $\left(\mathrm{TiO}_{2}\right)$ formada espontaneamente durante o momento do implante cresceu para 200nm após 5 anos. Isso significa que no organismo há uma liberação contínua de íon titânio como uma consequência dos constantes efeitos corrosivos. A camada espontânea de óxido não impede a passagem entre o metal e o organismo humano e gera uma estrutura porosa que pode atuar, inclusive, como um portador de vírus. Para se evitar esse fenômeno foi desenvolvido um procedimento onde uma camada de óxido de titânio anodizada é incluída sobre a superfície do implante após uma limpeza mecânica e química. Um tratamento térmico permite cristalinizar a camada oxidada, conferindo propriedades isolantes. A nova camada anodizada sobre o titânio isola o metal do organismo e previne que os íons metálicos migrem para dentro do organismo.

Algumas preocupações relacionadas ao uso desses metais são a atrofia óssea, a palpabilidade, o afrouxamento do dispositivo, a sensibilidade pela temperatura e interferência com terapia de radiação e diagnóstico por imagens. A permanente palpabilidade do titânio é frequentemente indesejada pelos pacientes, onde a interferência radiográfica pode diminuir a clareza de um estudo quando do acompanhamento de resultados neurocirúrgicos ${ }^{21,22}$. Em alguns casos, a remoção dos implantes de titânio é necessária. As razões para esse procedimento ocorrem por irritação crônica da pele ou dor crônica na região do implante ${ }^{23}$.

A revisão dos principais biomateriais utilizados para a reconstrução facial permite visualizar como os mesmos podem ser distribuídos através de implantes cirúrgicos e criar um mapa de distribuição dos materiais para uma rápida referência. Essa visão geral é apresentada na Figura 6, com destaque às áreas de aplicação de cada biomaterial.

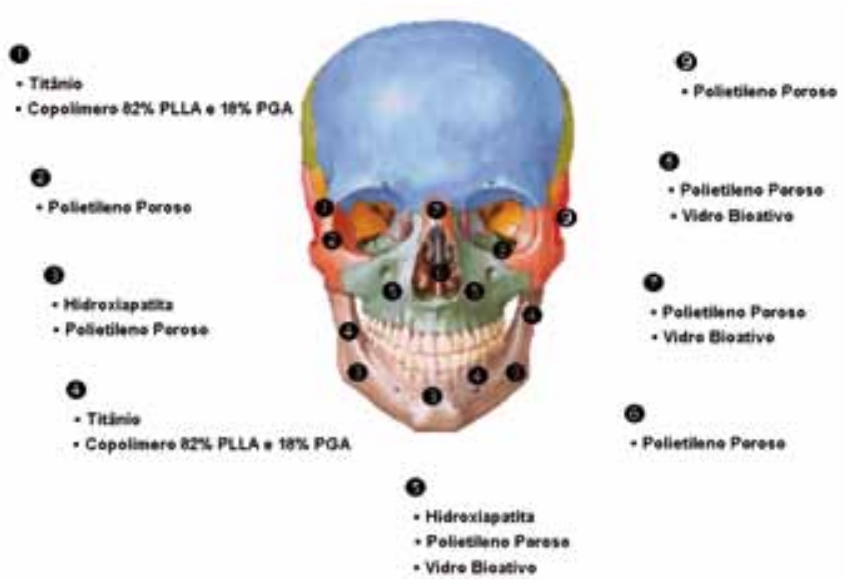

Figura 6 - Distribuição dos principais biomateriais para reconstrução da estrutura facial.

\section{CONCLUSÃO}

A utilização de biomateriais para a reconstrução facial em substituição aos materiais autogênicos ocorre em uma escala cada vez mais ampla. Apesar de existirem muitos biomateriais em uso, um critério na seleção para determinar a escolha dos biomateriais nos diversos tipos de reconstruções ainda não foi estabelecido, e o uso de biomateriais nas cirurgias faciais não é bem definido. Usualmente, a preferência pessoal é o fator determinante da escolha dos biomateriais. Quando o cirurgião conhece as propriedades exatas, as vantagens e as desvantagens e também como manusear os biomateriais nas reconstruções faciais, seu campo de atuação se expande na mesma proporção.

\section{REFERÊNCIAS}

1. Mitragotri S, Lahann J. Physical approaches to biomaterial design. Nat Mater. 2009;8(1):15-23.

2. Ziupos P. Recent development in the study of failure solid biomaterials and bone: 'fracture' and 'pre-fracture' toughness. Material Science and Engineering C. 1998;6(1):33-40.

3. Chim H, Gosain AK. Biomaterials in craniofacial surgery: experimental studies and clinical application. J Craniofac Surg. 2009;20(1):29-33.

4. Aitasalo KM, Peltola MJ. Bioactive glass hydroxyapatite in fronto-orbital defect reconstruction. Plast Reconstr Surg. 2007;120(7):1963-72.

5. Miles BA, Sinn DP, Gion GG. Experience with cranial implant-based prosthetic reconstruction. J Craniofac Surg. 2006;17(5):889-97.

6. Burg KJ, Porter S, Kellam JF. Biomaterial developments for bone tissue engineering. Biomaterials. 2000;21(23):2347-59.

7. Genecov DG, Kremer M, Agarwal R, Salyer KE, Barcelo CR, Aberman HM, et al. Norian craniofacial repair system: compatibility with resorbable and nonresorbable plating materials. Plast Reconstr Surg. 2007;120(6):1487-95.

8. Rah DK. Art of replacing craniofacial bone defects. Yonsei Med J. 2000;41(6):756-65

9. Cheung H, Lau K, Lu T, Hui D. A critical review on polymer-based bio-engineered materials for scaffold development. Composites Part B. 2007;38:291-300. 
10. Anderson JM, Rodriguez A, Chang DT. Foreign body reaction to biomaterials. Semin Immunol. 2008;20(2):86-100

11. Majewski WT, Yu JC, Ewart C, Aguillon A. Posttraumatic craniofacial reconstruction using combined resorsable and nonresorsable fixation systems. Ann Plast Surg. 2002;48(5):471-6.

12. http://3skimo.com/network_medical/ophthalmic_hydroxyapatite_implant.html

13. https://portal.m3-connect.de/wbs2/index.php?language=en

14. Gosain AK, Chim H, Arneja JS. Application-specific selection of biomaterials for pediatric craniofacial reconstruction: developing a rational approach to guide clinical use. Plast Reconstr Surg. 2009;123(1):319-30.

15. http://www.porexsurgical.com/english/surgical/skull.asp

16. Menderes A, Baytekin C, Topcu A, Yilmaz M, Barutcu A. Craniofacial reconstruction with high-density porous polyethylene implants. J Craniofac Surg. 2004;15(5):719-24

17. Burstein FD. Resorbable distraction of the mandible: technical evolution and clinical experience. J Craniofac Surg. 2008;19(3):637-43.
18. Dhol WS, Reyneke JP, Tompson B, Sándor GK. Comparison of titanium and resorbable copolymer fixation after Le Fort I maxillary impaction. Am J Orthod Dentofacial Orthop. 2008;134(1):67-73.

19. Suba C, Velich N, Turi C, Szabó G. Surface analysis methods of biomaterials used in oral surgery: literature review. J Craniofac Surg. 2005;16(1):31-6.

20. Jack JM, Stewart DH, Rinker BD, Vasconez HC, Pu LL. Modern surgical treatment of complex facial fractures: a 6-year review. J Craniofac Surg. 2005;16(4):726-31

21. Sanger C, Soto A, Mussa F, Sanzo M, Sardo L, Donati PA, et al. Maximizing results in craniofacial surgery with bioresorbable fixation devices. J Craniofac Surg. 2007;18(4):926-30.

22. Leonhardt H, Demmrich A, Mueller A, Mai R, Loukota R, Eckelt U. INION compared with titanium osteosynthesis: a prospective investigation of the treatment of mandibular fractures. Br J Oral Maxillofac Surg. 2008;46(8):631-4.

23. MylanusEA,JohanssonCB,Cremers CW.Craniofacialtitaniumimplants and chronic pain: histologic findings. Otol Neurotol. 2002;23(6):920-5..

\section{Correspondência para:}

Rua Major Lopes, 55 - 1501 - São Pedro - Belo Horizonte, MG, Brasil - CEP 30330-050

E-mail: mariomaia.plastica@gmail.com 